\title{
Implicit Equations of Non-degenerate Rational Bezier Quadric Triangles
}

\author{
Alicia Canton, L. Fernandez-Jambrina, E. Rosado Marıa, \\ and M.J. Vazquez-Gallo
}

\begin{abstract}
In this paper we review the derivation of implicit equations for non-degenerate quadric patches in rational $\mathrm{B}$ 'ezier triangular form. These are the case of Steiner surfaces of degree two. We derive the bilinear forms for such quadrics in a coordinate-free fashion in terms of their control net and their list of weights in a suitable form. Our construction relies on projective geometry and is grounded on the pencil of quadrics circumscribed to a tetrahedron formed by vertices of the control net and an additional point which is required for the Steiner surface to be a non-degenerate quadric.
\end{abstract}

\section{Introduction}

Bézier triangles [7] are an alternative to tensor product patches as an extension of the Bézier formalism from curves to surfaces. In fact they were already present in De Casteljau's original work. Though they are not widely used as tensor product patches, they are useful in finite element methods and in gaming and animation, since the triangular geometry is more versatile for building surfaces and avoids the formation of singular points.

Quadrics are extensively used in engineering and therefore a usual requirement for a design formalism is that it may represent quadrics in an exact fashion. Quadric patches can be described as rational quadratic Bézier triangles, though not every rational quadratic Bézier triangle is a quadric patch. A characterisation can be found in [2]. In general rational quadratic Bézier triangles are quartic surfaces known as Steiner surfaces. This family of surfaces includes ruled cubics and quadrics as subcases.

The relation between rational quadratic Bézier triangles and Steiner surfaces has been studied since the very beginning of CAGD. In [11] properties of Steiner surfaces are derived and they are postulated as candidates for surface design. In [9] a control polyhedron is used for representing quadric patches. The authors of [6] define a generalised stereographic projection on the sphere to derive results for 
quadratic and biquadratic patches. In [4] algebraic geometry is used for studying surfaces that can be parametrised quadratically. In [5] general Bézier triangles are studied as projections of Veronese surfaces and the quadratic case is classified.

In [1] algebraic geometry methods are used to determine whether a rational quadratic Bézier triangle is a quadric patch and an algorithm is provided for classifying them. A tool named Weighted Radial Displacement is proposed for constructing Bézier conics and quadrics in [10].

In this paper we address the calculation of implicit equations for nondegenerate quadrics in rational Bézier triangular form. Our goal is to find coordinate-free expressions that involve just the control net and weights for the patch, using algebraic projective geometry, as we did in [3]. This is useful, for instance, to compute geometric characteristics of the surfaces.

In Sect. 2 we review rational Bézier quadratic patches, introduce notation and define a pencil of quadrics through the corners of the control net of the patch and an additional point where the conics located on the boundary of the patch meet. In order to determine the coefficients of the pencil of quadrics, in Sect. 3 we derive an expression for the bilinear form of a conic circumscribed to a triangle in terms of its control points and weights. In Sect. 4 we show that the data we have from each boundary conic of the Steiner surface is compatible precisely if the surface is a quadric. In Sect. 5 we obtain the bilinear form for the Steiner quadric. Section 6 to several examples.

\section{Quadric Steiner Surfaces}

We consider rational Bézier quadratic triangles,

$$
c(u, v, w)=\frac{\sum_{i+j+k=2} \frac{2 !}{i ! j ! k !} \omega_{i j k} c_{i j k} u^{i} v^{j} w^{k}}{\sum_{i+j+k=2} \frac{2 !}{i ! j ! k !} \omega_{i j k} u^{i} v^{j} w^{k}}, \quad u+v+w=1, \quad u, w \in[0,1],
$$

defined by its control points, $\left\{c_{002}, c_{011}, c_{020}, c_{101}, c_{110}, c_{200}\right\}$, and their respective weights, $\left\{\omega_{002}, \omega_{011}, \omega_{020}, \omega_{101}, \omega_{110}, \omega_{200}\right\}$, which are real numbers.

Such surface patches are bounded by three curves, defined respectively by the equations $u=0, v=0, w=0$. For instance, the arc at $u=0$ is parametrised by

$$
c_{u}(v)=\frac{\sum_{j=0}^{2}\left(\begin{array}{l}
2 \\
j
\end{array}\right) \omega_{0 j 2-j} c_{0 j 2-j} v^{j}(1-v)^{2-j}}{\sum_{j=0}^{2}\left(\begin{array}{l}
2 \\
j
\end{array}\right) \omega_{0 j 2-j} v^{j}(1-v)^{2-j}}, \quad v \in[0,1],
$$

and hence it is a conic arc with control polygon $\left\{c_{002}, c_{011}, c_{020}\right\}$ and weights $\left\{\omega_{002}, \omega_{011}, \omega_{020}\right\}$. 


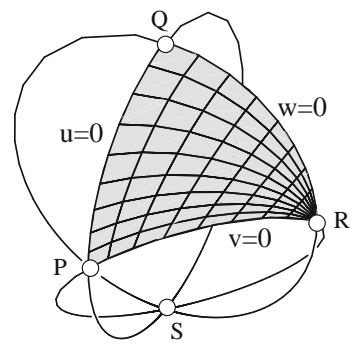

Fig. 1. Characterisation of quadric Steiner surfaces

Similarly, the conic arc at $v=0$ has control polygon $\left\{c_{002}, c_{101}, c_{200}\right\}$ and weights $\left\{\omega_{002}, \omega_{101}, \omega_{200}\right\}$, whereas the control polygon of the one at $w=0$ is $\left\{c_{020}, c_{110}, c_{200}\right\}$, with list of weights $\left\{\omega_{020}, \omega_{110}, \omega_{200}\right\}$. We assume from now on that these conics are non-degenerate.

The quadratic surface patch in (1) is generically a quartic surface, named Steiner surface [11], but in some particular cases it is a ruled cubic or a quadric. We are interested in the latter case due to the relevance of quadric surfaces. A characterisation of quadric Steiner surfaces is available in [2]:

- If the Steiner surface is a non-degenerate quadric, the three conic sections meet at a point $S$ and their tangents span a plane there (see Fig. 1).

- If the three conic sections meet at a point $S$ and their tangents span a plane there, the Steiner surface is a quadric.

The existence of point $S$ is useful for our purposes. We label the points at the corners of the surface patch as $P=c_{002}, Q=c_{020}, R=c_{200}$.

The three conic arcs defined by $u=0, v=0$ and $w=0$ are respectively located at planes that we denote $u, v, w$. We consider an additional plane $t$ through $P, Q, R$ (see Fig. 2).

In order to simplify the notation, we also call $t, u, v, w$ the linear forms associated to the respective planes. Since they are defined up to a constant, we fix them by requiring

$$
t(S)=u(R)=v(Q)=w(P)=1 .
$$

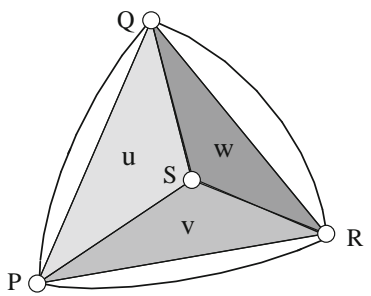

Fig. 2. Tetrahedron inscribed in a quadric Steiner surface 
Since a quadric is determined by nine independent conditions, the pencil of quadrics through $P, Q, R, S$ has five independent coefficients [12]. It is easy to check that the bilinear form $C$ for such pencil in a coordinate-free fashion is

$$
C=\lambda_{t u} t u+\lambda_{t v} t v+\lambda_{t w} t w+\lambda_{u v} u v+\lambda_{u w} u w+\lambda_{v w} v w,
$$

in terms of the linear forms for the planes containing the faces of the tetrahedron.

We have the bilinear form for the quadric except for the unknown coefficients. In the following section we determine the coefficients $\lambda_{i j}$ by restricting $C$ to the planes $u, v, w$. Since the intersection of the quadric with such planes are conics with known control polygons and weights, we determine the coefficients up to proportionality factors.

\section{Bilinear Forms for Conic Sections}

In order to determine the free coefficients of our pencil of quadrics, we need the bilinear forms for the conic sections of each of the faces of the tetrahedron. On Fig. 3 we have the conic on the face $u$ of the tetrahedron.

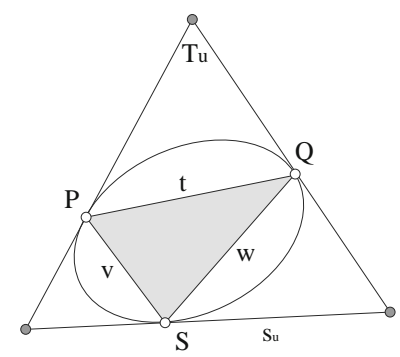

Fig. 3. Conic circumscribing a triangle

This conic has a bilinear form which is proportional to

$$
C_{u}=\lambda_{t v} t v+\lambda_{t w} t w+\lambda_{v w} v w .
$$

In this Section $t, v, w$ designate the straight lines which are the intersections of the respective planes with the plane $u$, as well as their linear forms. That is, for simplicity in the notation in this section, we identify $v$ with its restriction $\left.v\right|_{u}$ on the plane $u$.

The conic on $u$ is determined by noticing that the polar line of the control point $T_{u}=c_{011}$ is $t$, since this is the line linking the points $P, Q$ where the tangent lines from $T_{u}$ meet the conic arc. That is, $C_{u}\left(T_{u}, X\right)$ is proportional to $t(X)$ for all $X$ on the plane. For simplicity, we follow in most cases the following notation: the polar line of a point $A$ is the line $a$. 
This conic arc from $P$ to $Q$ is parametrised by

$$
c_{u}(t)=\frac{\omega_{002} P(1-t)^{2}+2 \omega_{011} T_{u} t(1-t)+\omega_{020} Q t^{2}}{\omega_{002}(1-t)^{2}+2 \omega_{011} t(1-t)+\omega_{020} t^{2}}, \quad t \in[0,1],
$$

but the weights are defined up to a Möbius transformation of the interval $[0,1]$ onto itselt [8],

$$
t(\tilde{t})=\frac{\tilde{t}}{(1-\rho) \tilde{t}+\rho}, \quad \tilde{t} \in[0,1],
$$

which produces a new list of weights for the same conic arc,

$$
\tilde{\omega}_{002}=\rho^{2} \omega_{002}, \quad \tilde{\omega}_{011}=\rho \omega_{011}, \quad \tilde{\omega}_{020}=\omega_{020} .
$$

We may use this degree of freedom to reparametrise the conic arc so that

$$
S=\lim _{\tilde{t} \rightarrow \infty} c_{u}(t(\tilde{t}))=\frac{\tilde{\omega}_{002} P-2 \tilde{\omega}_{011} T_{u}+\tilde{\omega}_{020} Q}{\tilde{\omega}_{002}-2 \tilde{\omega}_{011}+\tilde{\omega}_{020}},
$$

which has the advantage of writing the barycentric combination for $S$ in terms of the control polygon with coefficients that are simply the weights for the curve. Thus,

$$
T_{u}=\frac{\tilde{\omega}_{002} P+\left(2 \tilde{\omega}_{011}-\tilde{\omega}_{002}-\tilde{\omega}_{020}\right) S+\tilde{\omega}_{020} Q}{2 \tilde{\omega}_{011}},
$$

we can write the linear form for the polar line for $T_{u}$ in the frame $\{P, S, Q\}$ with respect to the conic $C_{u}$ as

$$
\begin{aligned}
C_{u}\left(T_{u}, X\right) & \propto\left(\lambda_{t v} \tilde{\omega}_{020}+\lambda_{t w} \tilde{\omega}_{002}\right) t(X)+\left(\lambda_{t v}\left(2 \tilde{\omega}_{011}-\tilde{\omega}_{002}-\tilde{\omega}_{020}\right)+\lambda_{v w} \tilde{\omega}_{002}\right) v(X) \\
& +\left(\lambda_{t w}\left(2 \tilde{\omega}_{011}-\tilde{\omega}_{002}-\tilde{\omega}_{020}\right)+\lambda_{v w} \tilde{\omega}_{020}\right) w(X),
\end{aligned}
$$

where the usual symbol $\propto$ means "proportional to".

Requiring that $t$ be the polar line of $T_{u}$, we get the unknown coefficients of its bilinear form,

$$
C_{u} \propto \tilde{\omega}_{002} t v+\tilde{\omega}_{020} t w+\left(\tilde{\omega}_{002}-2 \tilde{\omega}_{011}+\tilde{\omega}_{020}\right) v w .
$$

If $S$ were a point at infinity, instead of (6) we would need

$$
S=\tilde{\omega}_{002} P-2 \tilde{\omega}_{011} T_{u}+\tilde{\omega}_{020} Q \Rightarrow T_{u}=\frac{\tilde{\omega}_{002} P-S+\tilde{\omega}_{020} Q}{2 \tilde{\omega}_{011}},
$$

and we read the coefficients again imposing that $t$ is the polar line of $T_{u}$,

$$
C_{u} \propto \tilde{\omega}_{002} t v+\tilde{\omega}_{020} t w+v w .
$$

We can summarise this result in the following lemma: 
Lemma 1. The bilinear form for a conic circumscribed to a triangle PQS with sides $t, v, w$ as in Fig. 3 is

$$
\begin{cases}\tilde{\omega}_{002} t v+\tilde{\omega}_{020} t w+\left(\tilde{\omega}_{002}-2 \tilde{\omega}_{011}+\tilde{\omega}_{020}\right) v w, & \text { if } \tilde{\omega}_{002}-2 \tilde{\omega}_{011}+\tilde{\omega}_{020} \neq 0, \\ \tilde{\omega}_{002} t v+\tilde{\omega}_{020} t w+v w, & \text { if } \tilde{\omega}_{002}-2 \tilde{\omega}_{011}+\tilde{\omega}_{020}=0,\end{cases}
$$

where $S=\tilde{\omega}_{002} P-2 \tilde{\omega}_{011} T_{u}+\tilde{\omega}_{020} Q$ up to a constant.

The tangent line $s$ to the conic at $S$ is then $\tilde{\omega}_{002} v+\tilde{\omega}_{020} w$, with tangent vector $\boldsymbol{s}_{u}=\tilde{\omega}_{020} \overrightarrow{S P}-\tilde{\omega}_{002} \overrightarrow{S Q}$.

\section{Reparametrising the Quadric}

The latter theorem provides some of the unknown coefficients in (3) up to a constant. Since we have made use of a special choice of weights on $u$ to reach this result, we have to check that we can make it on the three boundary conics at a time in order to apply it to the whole quadric. We try to reparametrise the three boundary conics as in (6).

After reparametrising the conic on the plane $u$, the new list of weights is

$$
\left\{\rho^{2} \omega_{002}, \rho \omega_{011}, \omega_{020}, \rho \omega_{101}, \omega_{110}, \omega_{200}\right\},
$$

for some constant $\rho$ and we obtain a tangent vector $\boldsymbol{s}_{u}=\omega_{020} \overrightarrow{S P}-\rho^{2} \omega_{002} \overrightarrow{S Q}$ to the conic at $S$.

If we reparametrise the conic arc from $R$ to $Q$ on the plane $w$, the list of weights changes again,

$$
\left\{\rho^{2} \omega_{002}, \rho \omega_{011}, \omega_{020}, \sigma \rho \omega_{101}, \sigma \omega_{110}, \sigma^{2} \omega_{200}\right\},
$$

for some constant $\sigma$ and we get a new tangent vector $\boldsymbol{s}_{w}=\omega_{020} \overrightarrow{S R}-\sigma^{2} \omega_{200} \overrightarrow{S Q}$ to the conic at $S$.

Finally, if we needed to reparametrise the conic arc from $P$ to $R$ on the plane $v$, the list of weights would change to

$$
\left\{\tau^{2} \rho^{2} \omega_{002}, \tau \rho \omega_{011}, \omega_{020}, \tau \sigma \rho \omega_{101}, \sigma \omega_{110}, \sigma^{2} \omega_{200}\right\},
$$

for some constant $\tau$ and we would obtain another tangent vector $\boldsymbol{s}_{v}=$ $\sigma^{2} \omega_{200} \overrightarrow{S P}-\tau^{2} \rho^{2} \omega_{002} \overrightarrow{S R}$ to the conic at $S$.

The last reparametrisation obviously spoils the previous ones, but we may check whether it is necessary or not.

If the Steiner patch is a non-degenerate quadric, the three tangent vectors are to lie on a plane [2]. The determinant of these vectors,

$$
\operatorname{det}\left(\boldsymbol{s}_{u}, \boldsymbol{s}_{v}, \boldsymbol{s}_{w}\right)=\left|\begin{array}{ccc}
\omega_{020} & -\rho^{2} \omega_{002} & 0 \\
\sigma^{2} \omega_{200} & 0 & -\tau^{2} \rho^{2} \omega_{002} \\
0 & -\sigma^{2} \omega_{200} & \omega_{020}
\end{array}\right|=\rho^{2} \sigma^{2} \omega_{002} \omega_{020} \omega_{200}\left(1-\tau^{2}\right)
$$


tells us that they form a plane if and only if $\tau=1$, that is, the reparametrisations to locate $S$ at $t=\infty$ on the three conics are compatible.

From now on we omit the tildes over the weights, assuming that we are using a set of weights with this property,

$$
\begin{aligned}
S & =\frac{\omega_{002} c_{002}-2 \omega_{011} c_{011}+\omega_{020} c_{020}}{\omega_{002}-2 \omega_{011}+\omega_{020}}=\frac{\omega_{002} c_{002}-2 \omega_{101} c_{101}+\omega_{200} c_{200}}{\omega_{002}-2 \omega_{101}+\omega_{200}} \\
& =\frac{\omega_{200} c_{200}-2 \omega_{110} c_{110}+\omega_{020} c_{020}}{\omega_{200}-2 \omega_{110}+\omega_{020}}
\end{aligned}
$$

if $S$ is a point. If it is a point at infinity, $S$ has in principle three different representatives for each conic,

$$
\begin{gathered}
S_{u}=\omega_{002} c_{002}-2 \omega_{011} c_{011}+\omega_{020} c_{020}, \quad S_{v}=\omega_{002} c_{002}-2 \omega_{101} c_{101}+\omega_{200} c_{200}, \\
S_{w}=\omega_{200} c_{200}-2 \omega_{110} c_{110}+\omega_{020} c_{020},
\end{gathered}
$$

which are parallel vectors. We write the bilinear form for the conic on $u$ as

$$
\omega_{002} t v+\omega_{020} t w+t\left(S_{u}\right) v w
$$

to overcome this problem.

\section{Bilinear Forms for Steiner Quadrics}

If $S$ is a point, we have obtained bilinear forms for the conics on $u, v, w$ as

$$
\begin{aligned}
& C_{u} \propto \omega_{002} t v+\omega_{020} t w+\left(\omega_{002}-2 \omega_{011}+\omega_{020}\right) v w, \\
& C_{v} \propto \omega_{002} t u+\omega_{200} t w+\left(\omega_{002}-2 \omega_{101}+\omega_{200}\right) u w, \\
& C_{w} \propto \omega_{020} t u+\omega_{200} t v+\left(\omega_{020}-2 \omega_{110}+\omega_{200}\right) u v,
\end{aligned}
$$

and we can fit all pieces of information in the bilinear form:

Theorem 1. The bilinear form for a non-degenerate Steiner quadric patch, bounded by three non-degenerate conic arcs, with vertices of the control net $\left\{c_{002}, c_{011}, c_{020}, c_{101}, c_{110}, c_{200}\right\}$ and weights $\left\{\omega_{002}, \omega_{011}, \omega_{020}, \omega_{101}, \omega_{110}, \omega_{200}\right\}$, fulfilling that the intersection $S$ of the boundary conics is written as in (9) is

$$
\begin{aligned}
C & =\omega_{020} \omega_{002} t u+\omega_{002} \omega_{200} t v+\omega_{200} \omega_{020} t w+\omega_{002}\left(\omega_{020}-2 \omega_{110}+\omega_{200}\right) u v \\
& +\omega_{200}\left(\omega_{002}-2 \omega_{011}+\omega_{020}\right) v w+\omega_{020}\left(\omega_{002}-2 \omega_{101}+\omega_{200}\right) u w,
\end{aligned}
$$

where $u$ is the linear form of the plane containing $c_{002}, c_{011}, c_{020}$ which satisfies $u\left(c_{200}\right)=1, v$ is the linear form of the plane containing $c_{002}, c_{101}, c_{200}$ which satisfies $v\left(c_{020}\right)=1, w$ is the linear form of the plane containing $c_{020}, c_{110}, c_{200}$ which satisfies $w\left(c_{002}\right)=1$ and $t$ is the linear form of the plane containing $c_{002}, c_{020}, c_{200}$ which satisfies $t(S)=1$.

If $S$ is a point at infinity, the bilinear form is just

$$
\begin{aligned}
C & =\omega_{020} \omega_{002} t u+\omega_{002} \omega_{200} t v+\omega_{200} \omega_{020} t w \\
& +\omega_{002} t\left(S_{w}\right) u v+\omega_{200} t\left(S_{u}\right) v w+\omega_{020} t\left(S_{v}\right) u w .
\end{aligned}
$$




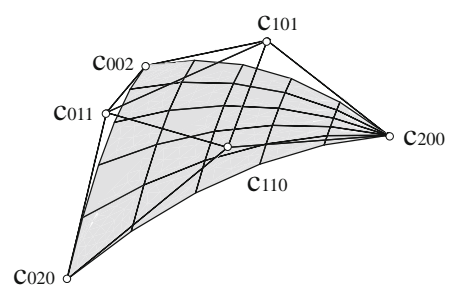

Fig. 4. Elliptic paraboloid

This result provides a procedure for computing a bilinear form for a nondegenerate Steiner quadric patch in a coordinate-free fashion using just the vertices of the control net and their respective weights:

1. Compute the normalised linear forms for the planes $t, u, v, w$.

2. Obtain $S$ as intersection of the planes $u, v, w$ and check if the patch belongs to a non-degenerate quadric.

3. Obtain an equivalent list of weights fulfilling (9).

4. Use Theorem 1 to obtain the bilinear form for the quadric patch.

5. The implicit equation for the quadric patch is then $C(X, X)=0$.

\section{Examples}

We use the previous results to compute implicit equations for several quadric patches:

Example 1. Net: $\left[\begin{array}{c}(0,0,0)(1,0,1)(2,0,0) \\ (0,1,1)(1,1,1) \\ (0,2,0)\end{array}\right]$ and weights: $\left[\begin{array}{ccc}1 & 1 & 1 \\ 1 & 1 \\ 1\end{array}\right]$ (Fig. 4):

The faces of the tetrahedron are the planes

$$
u: \frac{y}{2}=0, \quad v: \frac{x}{2}=0, \quad w: 1-\frac{x+y}{2}=0, \quad t:-\frac{z}{2}=0 .
$$

The planes $u, v, w$ meet at the point at infinity

$$
S=(0,0,-2)=c_{002}-2 c_{011}+c_{020}=c_{002}-2 c_{101}+c_{200}=c_{200}-2 c_{110}+c_{020} \text {. }
$$

The linear forms for the planes have been normalised according to (2). Hence the bilinear form for this surface is

$$
C=t u+t v+t w+u v+v w+u w
$$

and the implicit equation, in cartesian coordinates is

$$
0=\frac{2 x+2 y-x^{2}-y^{2}-x y-2 z}{4},
$$

which corresponds to an elliptic paraboloid. 


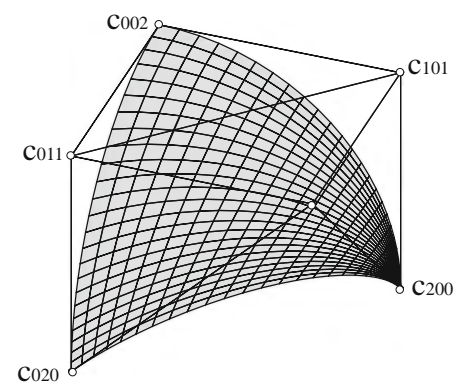

Fig. 5. Sphere

Example 2. Net: $\left[\begin{array}{c}(0,0,1)(1,0,1)(1,0,0) \\ (0,1,1)(1,1,1) \\ (0,1,0)\end{array}\right]$ and weights: $\left[\begin{array}{ccc}1 & 1 & 2 \\ 1 & 1 \\ 2\end{array}\right]$ (Fig. 5):

The faces of the tetrahedron are the planes

$$
u: y=0, \quad v: x=0, \quad w: \frac{1-x-y+z}{2}=0, \quad t: \frac{1-x-y-z}{2}=0 .
$$

The planes $u, v, w$ meet at the point $S=(0,0,-1)$. The bilinear form for this surface is

$$
C=2 t u+2 t v+4 t w+2 u v+2 v w+2 u w
$$

and the implicit equation in cartesian coordinates is

$$
0=1-x^{2}-y^{2}-z^{2},
$$

which corresponds to a sphere.

Example 3. Net: $\left[\begin{array}{c}(0,0,0)(1,0,0)(2,0,2) \\ (0,1 / 2,0) \\ (0,1,-1 / 2)\end{array}\right]$ and weights: $\left[\begin{array}{ccc}1 & 1 & 1 \\ 1 & 1 \\ 1\end{array}\right]$ (Fig. 6$)$ :

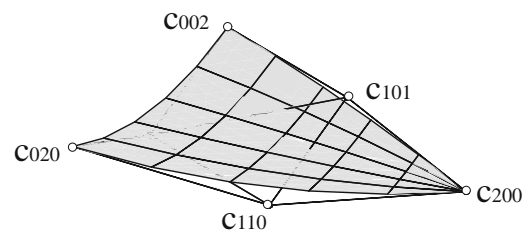

Fig. 6. Hyperbolic paraboloid 
The faces of the tetrahedron are the planes

$$
u: y=0, \quad v: \frac{x}{2}=0, \quad w: 1-\frac{x}{2}-y=0, \quad t: \frac{2 z-2 x+y}{4}=0 .
$$

The planes $u, v, w$ meet at the point at infinity

$$
S=(0,0,2)=c_{002}-2 c_{011}+c_{020}=-4\left(c_{002}-2 c_{101}+c_{200}\right)=\frac{4}{3}\left(c_{200}-2 c_{110}+c_{020}\right),
$$

and with the choice of bilinear form for $t$ we have

$$
t\left(S_{u}\right)=1, \quad t\left(S_{v}\right)=-\frac{1}{4}, \quad t\left(S_{w}\right)=\frac{3}{4} .
$$

Hence the bilinear form for this surface is

$$
C=t u+t v+t w+\frac{3}{4} u v+v w-\frac{1}{4} u w,
$$

and the implicit equation, in cartesian coordinates is

$$
0=\frac{2 z-x^{2}+y^{2}}{4}
$$

which corresponds to a hyperbolic paraboloid.

1. Albrecht, G.: Determination and classification of triangular quadric patches. Comput. Aided Geom. Des. 15(7), 675-697 (1998)

2. Boehm, W., Hansford, D.: Bézier patches on quadrics. In: Farin, G. (ed.) NURBS for Curves and Surface Design, pp. 1-14. SIAM (1991)

3. Cantón, A., Fernández-Jambrina, L., Rosado-María, E.: Geometric characteristics of conics in Bézier form. Comput. Aided Des. 43(11), 1413-1421 (2011)

4. Coffman, A., Schwartz, A.J., Stanton, C.: The algebra and geometry of steiner and other quadratically parametrizable surfaces. Comput. Aided Geom. Des. 13(3), 257-286 (1996)

5. Degen, W.: The types of triangular Bézier surfaces. In: Mullineux, G. (ed.) The Mathematics of Surfaces VI, pp. 153-170. Clarendon Press, Oxford (1996)

6. Dietz, R., Hoschek, J., Jüttler, B.: An algebraic approach to curves and surfaces on the sphere and on other quadrics. Comput. Aided Geom. Des. 10(3-4), 211-229 (1993)

7. Farin, G.: Triangular Bernstein-Bézier patches. Comput. Aided Geom. Des. 3(2), 83-127 (1986)

8. Farin, G.: Curves and Surfaces for CAGD: A Practical Guide, 5th edn. Morgan Kaufmann Publishers Inc., San Francisco (2002)

9. Lodha, S., Warren, J.: Bézier representation for quadric surface patches. Comput. Aided Des. 22(9), 574-579 (1990)

10. Sánchez-Reyes, J., Paluszny, M.: Weighted radial displacement: a geometric look at Bézier conics and quadrics. Comput. Aided Geom. Des. 17(3), 267-289 (2000)

11. Sederberg, T., Anderson, D.: Steiner surface patches. IEEE Comput. Graph. Appl. 5, 23-36 (1985)

12. Semple, J.G., Kneebone, G.T.: Algebraic Projective Geometry. Oxford University Press, London (1952) 